\title{
Fine-Tuning Nanoparticle Size by Oligo(guanine) $n$ Templated Synthesis of CdS: an AFM study
}

\author{
Lorenzo Berti*, Manuele Bellesia, Andrea Alessandrini and Paolo Facci \\ National Research Center on nanoStructures and bioSystems at Surfaces S3, \\ INFM-CNR, Via Campi 213/A, 41100 Modena, Italy
}

\section{Supporting Information}

\section{Materials and Methods}

Oligo $G_{n} S$ were purchased from Sigma Genosys (Madison, $\mathrm{WI}$ ). $\mathrm{CdCl}_{2}$ and $\mathrm{Na}_{2} \mathrm{~S}$ were purchased from Fluka (Buchs, Switzerland).

Millipore $18.2 \mathrm{M} \Omega / \mathrm{cm}$ water was used for preparing all solutions and obtained from a Millipore Simplicity 185 system (Billerica, MA).

AFM images were obtained with a Veeco Multimode (Santa Barbara, CA) equipped with a Nanoscope Illa controller used in tapping mode.

UV-Vis spectra were obtained with a Jasco (Easton, MD) V-550 UV-Vis spectrophotometer.

\section{Preparation of CdS}

In a typical experiment, oligo $G_{n}$ (where $n=5,10,15,20$ ) and $\mathrm{Cd}^{2+}$ were mixed at a 2:1 $\mathrm{G}: \mathrm{Cd}^{2+}$ ratio, and the mixture heated at $95{ }^{\circ} \mathrm{C}$ for $10 \mathrm{~min}$. Upon cooling, $\mathrm{S}^{2-}$ was added to obtain a final $\mathrm{Cd}^{2+}: \mathrm{S}^{2-}$ ratio of 1:10. The typical I volume of the resulting CdS suspension was $200 \mu \mathrm{L}$, with relative concentrations of $\left[\mathrm{Cd}^{2+}\right]=0.1 \mathrm{mM},[\mathrm{G}]=0.2 \mathrm{mM}$ and $\left[\mathrm{S}^{2-}\right]=1 \mathrm{mM}$. UV-Vis spectra were obtained after $24 \mathrm{~h}$.

\section{Preparation of AFM samples and imaging}

The colloidal CdS suspension prepared as described was diluted $1: 100$ in $\mathrm{ddH}_{2} 0$ and a $5 \mu \mathrm{L}$ aliquot deposited on freshly cleaved mica and air dried, followed by further drying under a gentle nitrogen flow.

Imaging was executed in tapping mode using a Multimode-Nanoscope Illa microscope (Veeco Instruments, Santa Barbara, CA) and TEST silicon cantilevers (resonance frequency $\sim 300 \mathrm{kHz}$ ). Imaging was performed for all the samples at the same oscillation frequency, free oscillation amplitude and setpoint amplitude of the cantilever.

\section{$\underline{\text { Particle size analysis }}$}


For each CdS population several AFM images were acquired. Such images were then flattened and planefitted using the Veeco sotfware algorithms. The images were then analyzed to obtain the nanoparticle height information. A threshold of $0.5 \mathrm{~nm}$ was applied in order to consider for statistical purposes only particles higher than $0.5 \mathrm{~nm}$. Applying this threshold avoids the introduction of artifacts, such as debris on the surface, to be included in the statistical analysis. A total of about 500 nanoparticles were considered for each oligo $\mathrm{G}_{\mathrm{n}}$ templated population. The data obtained from each oligo $G_{n}$ population yielded height distribution histograms from which Mean Height and Size Distribution were derived through a Gaussian Fit algorithm. The results of this statistical analysis are reported in Table S1.

TEM

Samples for TEM imaging were prepared by deposition of a concentrated aqueous CdS/DNA suspension on a carbon coated copper grid followed by air drying. TEM imaging was performed on a JEOL JEM 2010. EDAX microanalysis was executed with a Link INCA 100. 


\section{$\underline{\text { UV-Vis Spectra }}$}

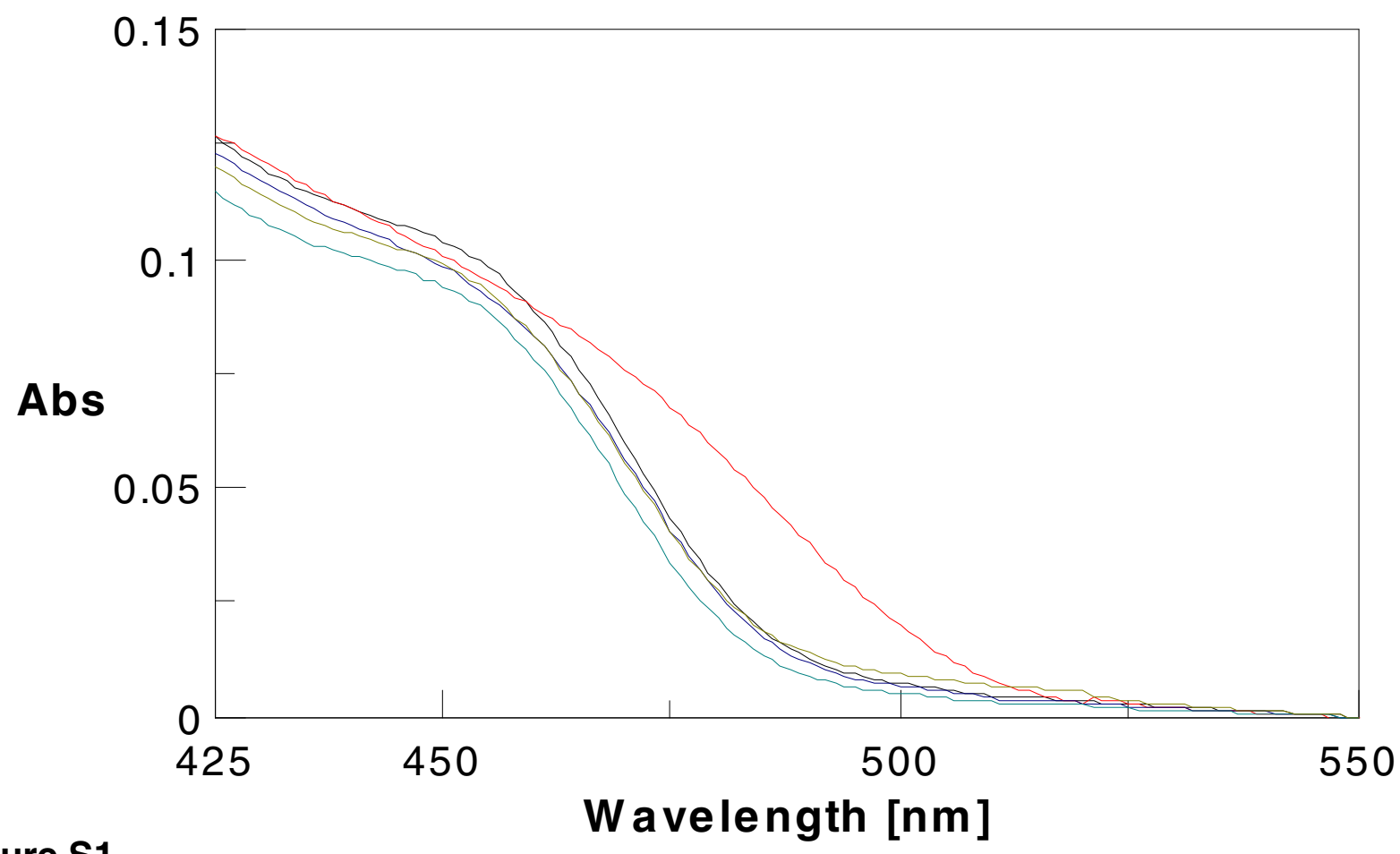

Figure S1

UV-Vis spectra of CdS formed without template (red line), and in the presence of oligoG $\mathrm{G}_{\mathrm{n}}$ of varying length after $24 \mathrm{~h}$ of aging. Quantum confinement in the presence of templating oligo $_{n}$ is witnessed by a steep blue-shifted absorption edge not present for bulk CdS (red line). However, direct comparison of the absorbtion spectra is not indicative of nanoparticle size differences as there is significant overlap between the differently capped CdS nanoparticles. 

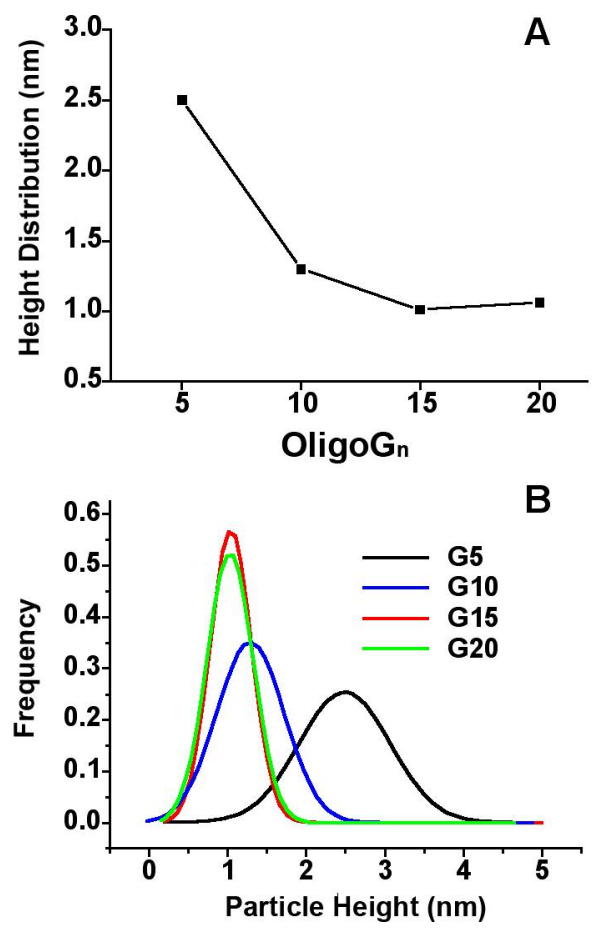

Figure S2.

(A) Average nanoparticle height dependence from oligo $G_{n}$ length; (B) Comparison of the normalized Gaussian fits for each CdS population. 


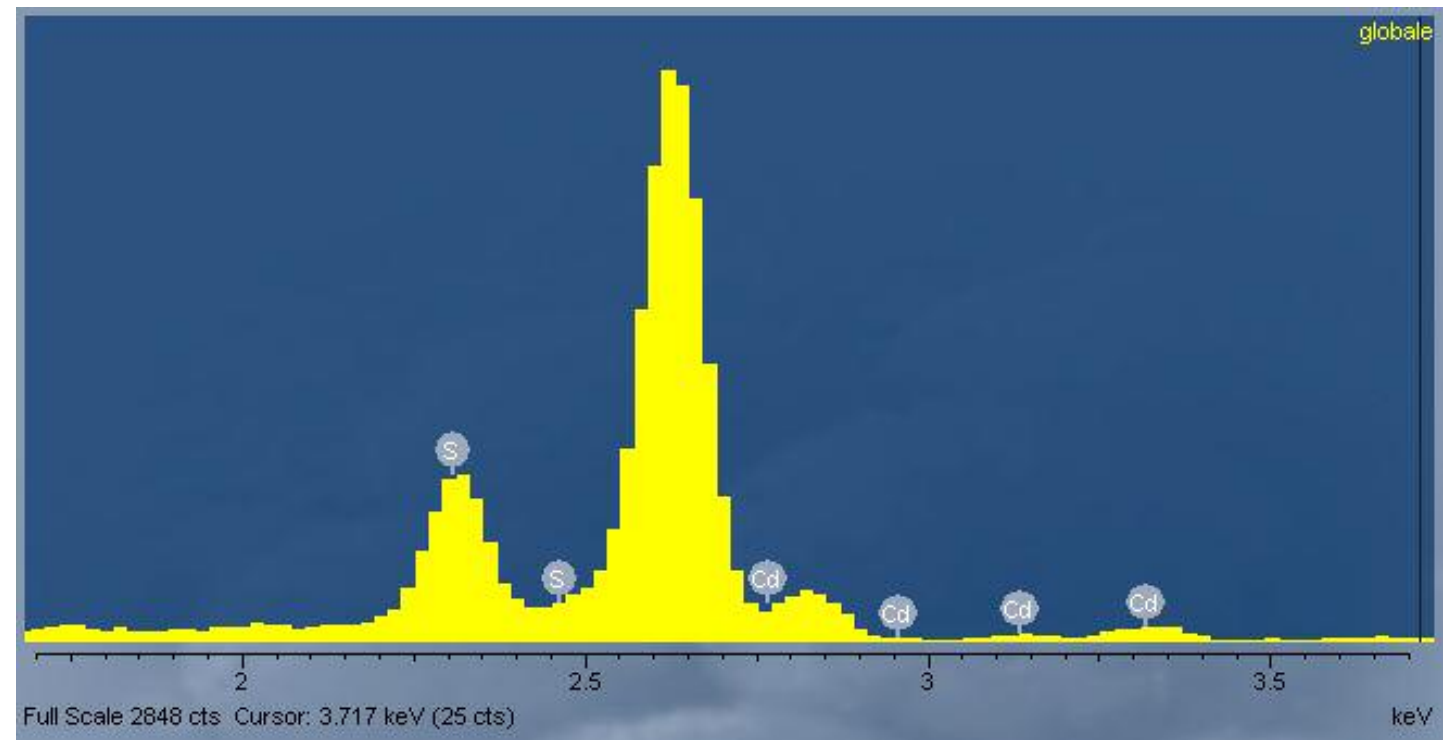

Figure S3.

EDAX micro analysis on a selected area containing CdS NPs. The position of the characteristic peaks for $\mathrm{Cd}$ and $\mathrm{S}$ are highlighted. The main peak located at the center of the spectrum is due to $\mathrm{Cl}$. 


\begin{tabular}{|c|c|}
\hline Sample & $\begin{array}{c}\text { Height }(\mathrm{nm}) \mathrm{t}=\mathbf{2 4 h} \\
\text { Gaussian fit }\end{array}$ \\
\hline oligoG $_{5}$ & $2.5 \pm 1.3$ \\
\hline oligoG $_{10}$ & $1.3 \pm 0.9$ \\
\hline oligoG $_{15}$ & $1.0 \pm 0.6$ \\
\hline oligoG $_{20}$ & $1.0 \pm 0.7$ \\
\hline
\end{tabular}

\section{Table S1}

Statistical data obtained by analyzing the height distribution from each oligo $G_{n}$-templated population 